\title{
Community Oncology and Rehabilitation
}

National Cancer Institute

\section{Source}

National Cancer Institute. Community Oncology and Rehabilitation. NCI Thesaurus. Code C16047.

Introduction, application, and evaluation of effective and practical cancer prevention, control, and rehabilitation intervention programs in community settings. 